\title{
A Case Series of Symptomatic Distal Biceps Tendinopathy
}

\author{
Jung Hyun Lee, Kyung Chul Kim, Ji-Ho Lee, Kee Baek Ahn, In Hyeok Rhyou \\ Department of Orthopaedic Surgery, Upper Extremity and Microsurgery Center, Pohang Semyeong Christianity Hospital, Pohang, Korea
}

Background: The study of conservative and surgical treatment of distal biceps tendinopathy and associated biceps tendon partial rupture. Methods: Twenty-one cases with distal biceps tendonitis and partial ruptures were studied who visited Pohang Semyeong Christianity Hospital from June 2010 to August 2017. The mean age was 57.1 years (39-69 years), 14 males and 7 females. The mean duration of symptom at the time of first visit was 4.9 months (0.2-14 months). Ultrasonography and magnetic resonance imaging were performed for patients with severe symptoms. According to the severity of the symptoms, splint immobilization, oral nonsteroidal anti-inflammatory drugs, and ultrasound-guided steroid injection were performed. Surgical treatment was performed if the patient did not respond to conservative treatment for 3 to 6 months or longer.

Results: There were 9 cases of partial rupture of the distal biceps tendon associated with distal biceps tendinopathy on imaging studies. Conservative treatment showed symptomatic improvement in 16 of 21 cases. In 4 cases with a relatively mild symptom, anti-inflammatory analgesics and intermittent splinting showed good result. In 12 cases, symptoms improved after ultrasonography-guided steroid injection. Surgical treatment was performed on 5 cases that did not respond to conservative treatment.

Conclusions: Conservative treatment of distal biceps tendinopathy may promise good results. However, in case of partial tear of the distal biceps tendon and refractory to conservative treatment, surgical treatment may be needed.

(Clin Shoulder Elbow 2018;21(4):213-219)

Key Words: Biceps tendon; Partial tear; Tendinopathy; Magnetic resonance imaging; Surgery

\section{Introduction}

The biceps brachii is an important muscle responsible for flexion and supination of the elbow joint, and a lesion of the biceps brachii leads to a decrease in the strength of flexion and supination of the elbow joint and causes pain during the movements. ${ }^{1)}$ The pathophysiology and clinical course of proximal biceps tendinopathy, which is a major cause of shoulder pain, is relatively well known, but the incidence of distal biceps tendinopathy is lower than that of proximal biceps tendinopathy, and its pathophysiology is not well known.

Distal biceps tendinopathy should be understood not as a single disease but as a spectrum of disease encompassing a group of disorders classified according to the site and severity of the lesion, such as bicipitoradial bursitis, distal biceps chronic tendon degeneration, distal biceps partial tear, and distal biceps complete tear. ${ }^{2,3)}$ The cases of complete tear of the distal biceps tendon are reported to account for $3 \%$ of the total cases of biceps tendon ruptures, and the prevalence of this disease is known to be 2.55 per 100,000 population. ${ }^{4)}$ With respect to the complete tear of the distal biceps tendon, many studies have reported that good outcomes can be obtained by surgical treatment rather than conservative treatment. ${ }^{4-6)}$ Most cases of distal biceps tendinopathy without the complete tear of the biceps tendon are generally associated with minor trauma or repeated activities without accompanying trauma. The severity of symptoms of distal biceps tendinopathy without an accompanying complete tear is variable, and the actual prevalence and natural

Received June 16, 2018. Revised August 28, 2018. Accepted September 3, 2018.

Correspondence to: In Hyeok Rhyou

Department of Orthopaedic Surgery, Upper Extremity and Microsurgery Center, Pohang Semyeong Christianity Hospital, 351 Posco-daero, Namgu, Pohang 37816, Korea

Tel: +82-54-289-1765, Fax: +82-54-289-1766, E-mail: osdrrih@gmail.com, ORCID: https://orcid.org/0000-0001-9312-2952

IRB approval: Pohang Semyeong Christianity Hospital (No. PSMCHIRB-18-05).

Financial support: None. Conflict of interests: None. 
course are not well known and the treatment methods are not well established. The purpose of this study was to evaluate the treatment outcomes of distal biceps tendinopathy with symptoms according to treatment methods.

\section{Methods}

In this study, we studied 21 patients who were diagnosed with distal biceps tendinopathy based on plain radiographic and magnetic resonance imaging (MRI) findings among the patients who came to our hospital and presented with elbow pain as the main complaint from June 2010 to August 2017.

The patients who were clinically suspected to have distal biceps tendinopathy but did not require additional imaging tests because they had mild symptoms or they responded well to conservative treatment and those for whom surgical procedures were primarily performed due to MRI findings of the complete tear of the distal biceps tendon were excluded from this study.

The patients complained mainly of pain in the cubital fossa and subjective decrease in the strength of flexion of the elbow joint and supination power of the forearm. On physical examination, patients complained of tenderness at the distal biceps tendon attachment site and pain during active elbow flexion and forearm supination. At their first visit to the hospital, the range of motion (ROM) of the elbow joint was measured and the scores of the pain visual analogue scale were recorded. At 6 months after performing each treatment, the degrees of pain and satisfaction were measured using the pain visual analogue scale and satisfaction rating scale (very poor, poor, good, very good, and excellent). The assessments of pain and satisfaction by the pain visual analogue scale and satisfaction rating scale were conducted by the questionnaire survey during the outpatient visits (13 cases), but when patients had difficulty coming to the hospital, the assessments were performed through telephone interviews (8 cases).
Using T1 and T2 fat-suppression views of the MRI of the elbow, we evaluated the presence or absence of the rupture of the distal biceps tendon attached to the radial tuberosity and detected the presence or absence of inflammatory findings around the tendon.

A patient was diagnosed with tendinitis when only peritendinous bursitis was observed without the intratendinous signal change of the distal biceps tendon. The cases where tendon enlargement or abnormal contour was observed and an abnormal intratendinous signal change of the distal biceps tendon was also detected were classified as partial tears (Fig. 1). In the cases of complete tears, discontinuity of the tendon was observed, and in the cases where even the biceps aponeurosis was ruptured, retraction of the tendon was found. ." When a complete tear was detected, surgical treatment was performed and the cases were excluded from this study.

For the patients diagnosed with distal biceps tendinopathy based on clinical and MRI findings, conservative treatment was first performed and a long arm splint was applied for 3 weeks in a forearm supination posture. In addition, physical therapy was performed while administering nonsteroidal anti-inflammatory drugs (NSAIDs).

Sono-guide steroid injection was performed when the symptoms were not improved despite the use of splinting for more than 3 weeks. Sono-guide steroid injection was performed after making the radial tuberosity turn toward the dorsal side of the forearm by full pronation of the forearm. After finding the exact location of the radial tuberosity by ultrasound imaging, a mixture of $2 \mathrm{mg}$ of ropivacaine, $40 \mathrm{mg}$ of triamcinolone, and $1 \mathrm{ml}$ of normal saline was injected into the tendon sheath.

Surgical treatment was performed for patients who did not respond to conservative treatment for 6 months or more. Surgery was performed by three orthopedic surgeons and was done by the anterior approach or two incision approach according to the preferences. Both methods removed the degenerative por-
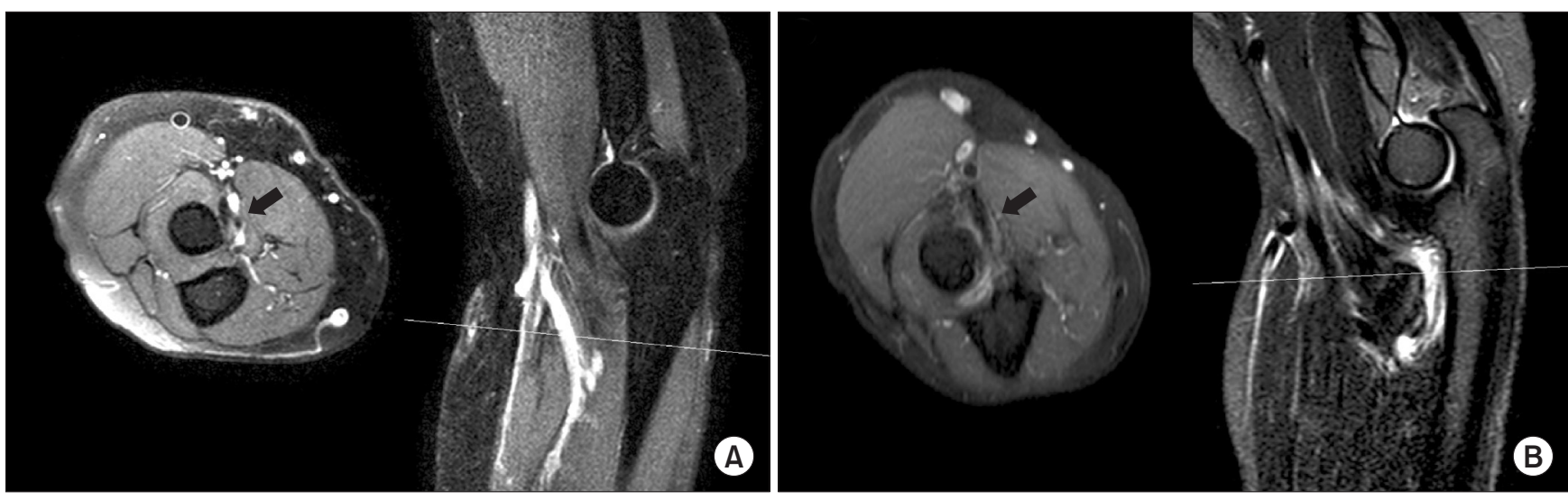

Fig. 1. Magnetic resonance imaging findings of distal biceps tendon. (A) Partial tear: tendon enlargement with abnormal contour, intratendinous signal intensity (arrow). (B) Tendinitis or peritendinous bursitis: abnormal signal intensity around distal biceps tendon (arrow). 
tion of the tendon tissue after tear completion of the partial tear of the distal biceps tendon and reattached it to the anatomical attachment site of the radial tuberosity. When the anterior approach was used (Fig. 2), an oblique skin incision of the site of the cubital fossa was made, the distal biceps tendon was completely separated from the attachment site, the degenerative abnormal area was removed, and the tendon was reattached to the anatomical site using the suture anchor. In the case of the two incision approach (Fig. 3), we first completely dissected the partial tear area of the distal biceps tendon by the anterior approach, excised the degenerated tendon, and performed the suture of the tendon. Then, passing the K-wire through the skin to create a bone tunnel at the attachment site of the biceps tendon, the posterior skin incision site was determined and the tendon was reattached by the pull-out suture technique. A long arm cast was applied in the position of forearm supination and $90^{\circ}$ elbow flexion for 6 weeks after surgery. Passive and active assisted ROM began at 6 weeks postoperatively followed by
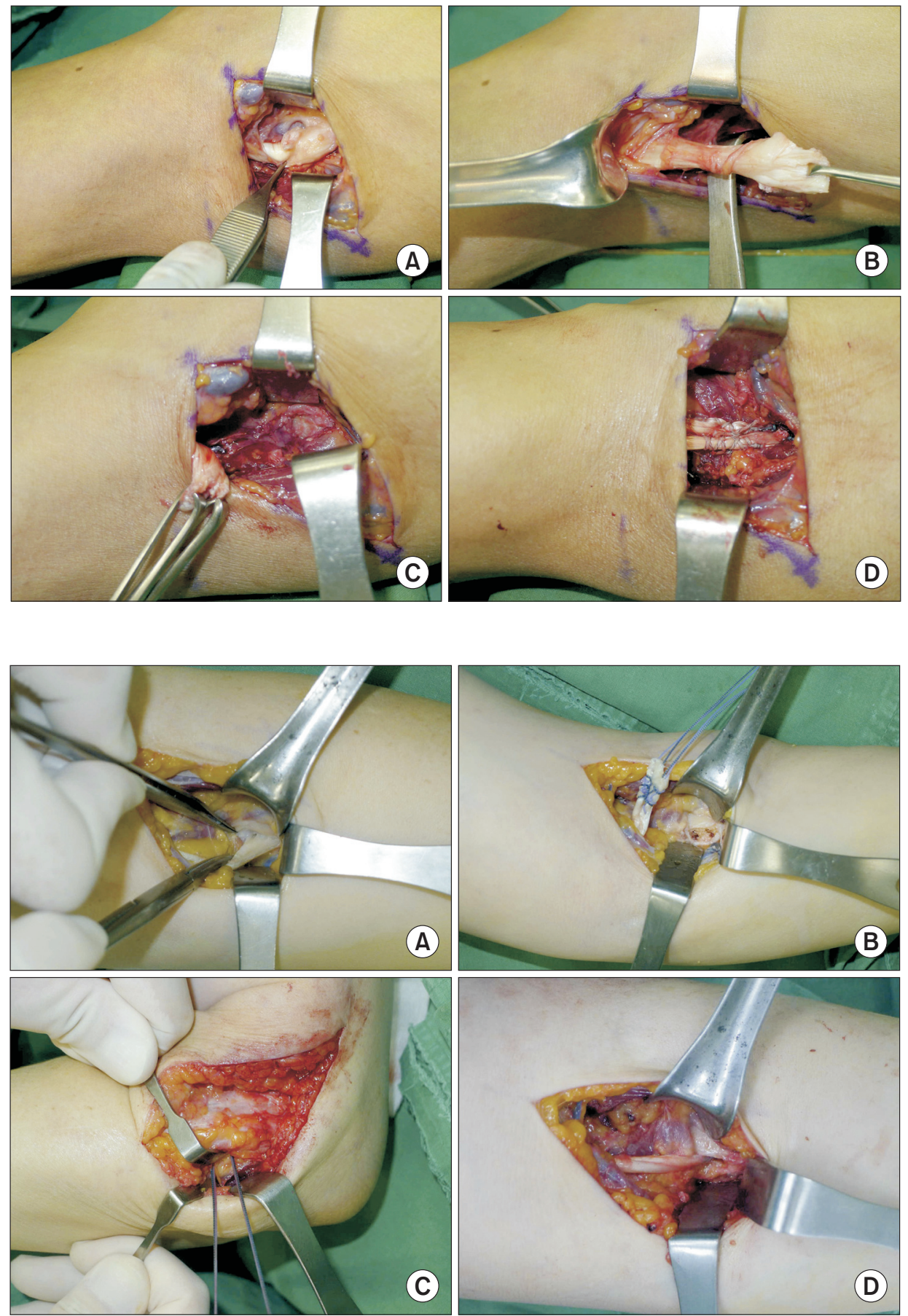

Fig. 2. Anterior approach to distal biceps tendon. (A) The partial tear of the tendon was observed at the distal biceps origin insertion site. (B) Completion of distal biceps tendon tear completion. (C) Debridement and decortication of radial tuberosity. (D) Reattachment with suture anchor.

3. Two incision approach to distal biceps tendon. (A) The partial tear of the tendon was observed at the distal biceps origin insertion site. (B) Tear completion, tendon preparation with fiberwire suture, radial tuberosity decortication. Two drill hole for pullout suture was made. (C) suture pulled out to posterior side of elbow. (D) Biceps tendon reattached anatomically. 
flexion and supination strengthening exercise was performed at 3 months after surgery.

\section{Results}

The average age of the patients was 57.1 years (39 to 69 years), and they consisted of 14 males and 7 females. The average duration of illness of the patients was 4.9 months ( 0.2 to 14 months) (Table 1). All the patients complained of pain during active flexion and active supination of the elbow joint and had tenderness at the insertion site of the distal biceps tendon. No patients had limitation of the ROM of the elbow compared to the opposite side.

On preoperative plain radiography and $\mathrm{MRI}$, a partial tear accompanied by distal biceps tendinitis was detected in 9 out of 21 cases $(42.9 \%)$, and calcification around the radial tuberosity accompanied by biceps tendinitis was observed in 2 cases (9.5\%). In the remaining 10 cases $(47.6 \%)$, no intratendinous signal change of the distal biceps tendon was detected, and only peritendinous bursitis was observed.
Sixteen out of 21 cases (76.2\%) showed symptomatic improvement only by conservative treatment, and the average time from the start of treatment to symptom improvement was 6.2 weeks ( 3 weeks to 3 months). Among the 16 cases, in 4 patients who showed MRI findings of only peritendinous bursitis and had mild symptoms, symptomatic improvement was achieved only by administration of NSAIDs and intermittent splinting. In 12 patients, symptoms were relatively severe and they were not improved only by application of plinting and administration of oral NSAIDs, so sono-guide steroid injection was performed, resulting in the improvement of symptoms (Fig. 4).

For the remaining five patients (23.8\%), who did not respond to splinting and sono-guide steroid injections, surgical treatment was performed. Of the five patients, three underwent surgery by the anterior approach and two did by the two incision approach. All of the five patients who required surgical treatment had a partial tear on MRI.

After surgical treatment, in 4 cases, tenderness and pain disappeared at 6 weeks to 3 months; in other words, tenderness and pain disappeared at 6 weeks in 2 cases and at 3 months in

Table 1. Summary of Patient Demographic, MRI Findings, Treatment, and Outcomes

\begin{tabular}{|c|c|c|c|c|c|c|c|c|}
\hline $\begin{array}{l}\text { Patient } \\
\text { No. }\end{array}$ & Sex & Age (yr) & MRI finding & Final Tx & $\begin{array}{l}\text { Period before Tx } \\
\text { (mo) }\end{array}$ & $\begin{array}{c}\text { Preoperative } \\
\text { pain VAS }\end{array}$ & $\begin{array}{l}\text { Post Tx } 6 \text { months } \\
\text { Pain VAS }\end{array}$ & $\begin{array}{l}\text { Satisfaction } \\
\text { rating scale }\end{array}$ \\
\hline 1 & $\mathrm{~F}$ & 53 & Partial tear & Surgery (two incision approach) & 8 & 7 & 5 & Poor \\
\hline 2 & $\mathrm{~F}$ & 50 & Tendinitis & Injection & 1 & 6 & 2 & Very good \\
\hline 3 & $\mathrm{M}$ & 57 & Partial tear & Surgery (anterior approach) & 6 & 4 & 2 & Very good \\
\hline 4 & M & 60 & Tendinitis & Injection & 7 & 5 & 3 & Good \\
\hline 5 & M & 39 & Partial tear & Surgery (anterior approach) & 14 & 6 & 2 & Good \\
\hline 6 & $\mathrm{~F}$ & 51 & Calcification & Splint \& medication & 7.5 & 6 & 2 & Very good \\
\hline 7 & M & 47 & Partial tear & Splint \& medication & 2 & 6 & 2 & Very good \\
\hline 8 & M & 56 & Partial tear & Injection & 5 & 7 & 1 & Excellent \\
\hline 9 & $\mathrm{M}$ & 62 & Partial tear & Surgery (anterior approach) & 7 & 8 & 1 & Good \\
\hline 10 & $\mathrm{~F}$ & 63 & Partial tear & Injection & 0.7 & 8 & 3 & Good \\
\hline 11 & $\mathrm{M}$ & 46 & Tendinitis & Injection & 4.5 & 6 & 2 & Very good \\
\hline 12 & M & 63 & Tendinitis & Injection & 3.5 & 7 & 2 & Very good \\
\hline 13 & M & 63 & Tendinitis & Injection & 5.5 & 6 & 2 & Very good \\
\hline 14 & M & 64 & Partial tear & Surgery (two incision approach) & 6 & 8 & 2 & Very good \\
\hline 15 & $\mathrm{~F}$ & 62 & Tendinitis & Injection & 3.5 & 7 & 1 & Excellent \\
\hline 16 & M & 63 & Partial tear & Injection & 0.2 & 6 & 2 & Very good \\
\hline 17 & $\mathrm{M}$ & 59 & Tendinitis & Injection & 6 & 6 & 2 & Very good \\
\hline 18 & M & 69 & Tendinitis & Injection & 6 & 4 & 2 & Very good \\
\hline 19 & $\mathrm{~F}$ & 50 & Tendinitis & Injection & 7 & 6 & 3 & Good \\
\hline 20 & $\mathrm{~F}$ & 58 & Calcification & Splint \& medication & 2 & 6 & 2 & Very good \\
\hline 21 & $\mathrm{M}$ & 65 & Tendinitis & Splint \& medication & 1 & 4 & 2 & Very good \\
\hline
\end{tabular}

MRI: magnetic resonance imaging, Tx: treatment, VAS: visual analogue scale, M: male, F: female. 
2 cases. In one case, although there was a slight improvement compared with preoperative pain, the patient complained of tenderness and pain even after 6 months postoperatively (Table 1,2).

Baseline (pre-treatment) Pain VAS scores were 5.5 points for the splint group, 6.2 points for the sono-guide steroid injection group, and 6.6 points for the surgery group and they improved to 2.0, 2.1, and 2.4 points, respectively at 6 months after treatment. Regarding the level of satisfaction assessed by the satisfaction rating scale at the final follow-up, the outcome of treatment was rated as very good by all 4 patients in the splint group, and it was rated as good by 3 patients, very good by 7 patients, and excellent by 2 patients in the sono-guide steroid injection group. It was rated poor by 1 patient, good by 2 patients, and very good by 2 patients in the surgery group (Table 3 ). There were no major postoperative complications such as radial nerve palsy and radioulnar synostosis after surgical treatment.

In 12 cases (57.1\%) without any MRI findings of tears, symptoms improved by splinting, medication or steroid injections without surgical treatment, but of the 9 cases (42.9\%) with partial tears in MRI findings, 4 cases improved in conservative treatment only, the remaining 5 cases required surgical treatment to improve symptoms.

The mean duration of illness before each treatment was 3.1 months in the splint group, 4.2 months in the steroid injection group, and 8.2 months in the surgery group.

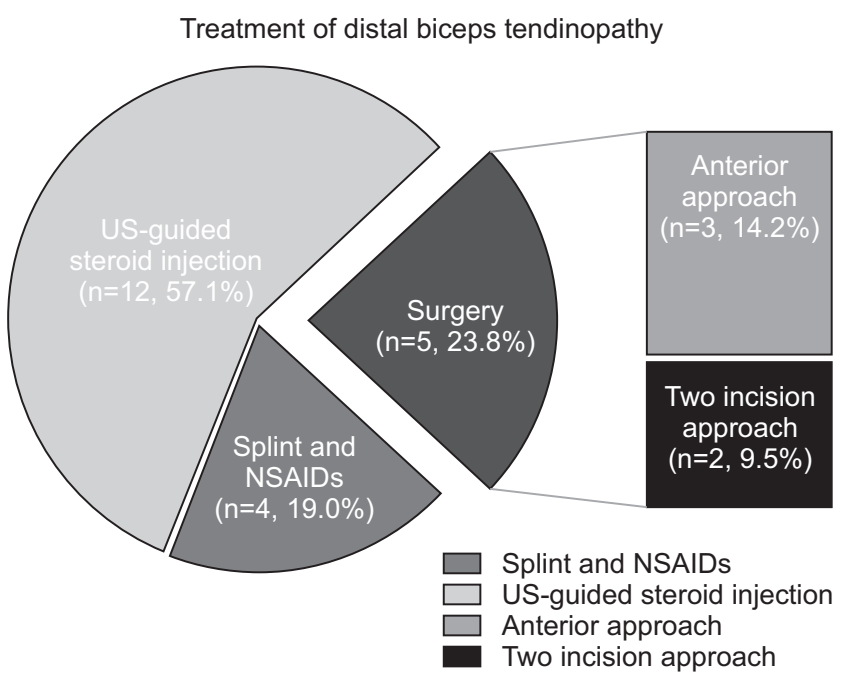

Fig. 4. Treatment options of distal biceps tendinopathy. Of 21 patients, 4 patients $(19.0 \%)$ showed symptomatic improvement only by medication and splinting, and 12 patients (57.1\%) showed improvement after ultrasound (US)-guided steroid injection. The remaining 5 patients (23.8\%) required surgical treatment.

NSAIDs: nonsteroidal anti-inflammatory drugs.

\section{Discussion}

Distal biceps tendinopathy has a wide disease spectrum and diverse symptoms. The precise prevalence is not known, and the treatment methods and natural course of this disease are not well known, either. If the patient is asymptomatic or has mild symptoms, he or she does not need any special treatment. If symptoms are present, oral NSAIDs or intermittent immobilization may be helpful, and extracorporeal shock wave therapy or sono-guide steroid injection may also be a good treatment option. ${ }^{7,8)}$

In this study, among nine patients $(42.9 \%)$ who were found to have a partial tear on MRI, five patients eventually required surgical treatment. Previous studies have shown that the patients with partial tears of distal biceps tendon may not respond well to conservative treatment. ${ }^{3,9-11)}$ Bain et al. ${ }^{11)}$ reported that conservative treatment frequently failed and surgical treatment was required in the cases accompanied by the partial tear of $50 \%$ or more of the distal biceps tendon. However, this result was obtained by examining the degrees of the tears using an endoscope during surgery. With respect to the effectiveness of MRI for biceps tendon ruptures, Festa et al. ${ }^{12)}$ pointed out that it is not easy to make a diagnosis of partial tears of the distal biceps tendon only with $\mathrm{MRI}$, reporting that while the sensitivity and specificity of MRI for complete tears were $100 \%$ and $82.8 \%$, respectively, MRI had a sensitivity of $59.1 \%$ and a specificity of $100 \%$ for partial tears. For accurate MRI testing of the distal bi-

Table 2. Pain VAS, Preoperative and Post Treatment

\begin{tabular}{ccc}
\hline & \multicolumn{2}{c}{ Pain VAS } \\
\cline { 2 - 3 } Group $^{*}$ & Preoperative & Post treatment $^{\dagger}$ \\
\hline Spint (4 cases) & 5.5 & 2.0 \\
Injection (12 cases) & 6.2 & 2.1 \\
Surgery (5 cases) & 6.6 & 2.4 \\
\hline
\end{tabular}

VAS: visual analogue scale.

*Spint: long arm splint at forearm supination position, Injection: sono-guided steroid injection, Surgery: tear completion and anatomical reattachment. ${ }^{\dagger} 6$ months after specific treatment.

Table 3. Satisfaction Rating Scale, 6 Months after Specific Treatment

\begin{tabular}{lcccccc}
\hline \multirow{2}{*}{ Group } & \multicolumn{5}{c}{ Satisfaction rating scale } \\
\cline { 2 - 7 } & Very poor & Poor & Good & Very good & Excellent & Total \\
\hline Spint (4 cases) & - & - & - & 4 & - & 4 \\
$\begin{array}{l}\text { Injection } \\
(12 \text { cases })\end{array}$ & - & - & 3 & 7 & 2 & 12 \\
$\begin{array}{c}\text { Surgery } \\
(5 \text { cases })\end{array}$ & - & 1 & 2 & 2 & - & 5 \\
\hline
\end{tabular}

*Spint: long arm splint at forearm supination position, Injection: sono-guided steroid injection, Surgery: tear completion and anatomical reattachment. 
ceps brachii tendon, Bain et al. ${ }^{11)}$ suggested that MRI should be performed in the position of shoulder abduction, elbow flexion, and forearm supination. In this study, MRI was taken in the conventional positions. In this regard, accurate MRI testing methods are also considered important to conduct comparative studies of treatments and rupture degrees of the biceps brachii tendon in the future. Although it was reported that good outcomes were achieved with debridement alone in some cases, ${ }^{9}$ surgical treatment of distal biceps tendinopathy is generally performed by anatomical reattachment after tear completion, and surgical approaches are largely divided into the anterior approach and bilateral incision approach., ${ }^{3,13-15)}$ When surgery is performed by the anterior approach, radial nerve palsy may occur as a result of excessive traction to secure a field of view of the surgical site, and it is difficult to accurately reattach the biceps tendon to the anatomical attachment site of the radial tuberosity. In the case of the two incision approach, anatomical reattachment is relatively easier and the risk of radial nerve palsy is lower. However, if the ulnar bone is excessively exposed during the process of approaching and dissecting it, radioulnar synostosis may occur. Therefore, caution is needed to avoid damaging or exposing the periosteum of the proximal ulna during dissection. ${ }^{9,16)}$ Bisson et al. ${ }^{17)}$ reported the results of surgical treatment of 45 cases of the complete tear of the distal biceps tendon, and stated that $27 \%$ of the subjects had postoperative complications, referring to radial nerve injury (15\%) and radioulnar synostosis $(7 \%)$ as the two most common types of complications. Regarding the postoperative rehabilitation, they recommended using an elbow hinge brace, allowing only passive flexion, pronation, and supination of $90^{\circ}$ or more from the day of surgery (day 0 ) after surgery, not allowing extension and active flexion, gradually increasing the range, and progressively starting strengthening at 6 weeks after surgery. However, in this study, elbow joint exercises were performed after immobilization with a long arm cast for 6 weeks. No quantitative measurement of the ROM was performed, but no patients complained of limited joint mobility after surgery.

One of the limitations of this study is that we did not deal with the natural course of the entire group of patients with distal biceps tendinopathy. Among the patients diagnosed clinically with distal biceps tendinopathy, only the patients for whom objective imaging tests, such as MRI were conducted because of a long duration of the disease or relatively severe symptoms were included in this study. Since the patients with mild or moderate symptoms were excluded from this study, this study has limitations on describing the natural courses of the entire group of patients. However, it is considered a meaningful outcome of this study that we suggested a treatment option to improve the clinical symptoms and the quality of life of patients by surgical treatment and obtained positive outcomes in the patients whose symptoms were not improved by means of conservative treatment. We believe further studies are needed to improve the un- derstanding of distal biceps tendonopathy, including pathologic changes and natural course of the disease.

\section{Conclusion}

In most cases of distal biceps tendinopathy, good results can be achieved by conservative treatment. However, the findings of this study suggest that surgical treatment can be an effective treatment option leading to the improvement of clinical symptoms in the cases where no symptomatic improvement can be achieved by long-term conservative treatment or the disease is accompanied by a partial tear of the distal biceps tendon.

\section{References}

1. Chew ML, Giuffrè BM. Disorders of the distal biceps brachii tendon. RadioGraphics. 2005;25(5):1227-37.

2. Kegels L, Van Oyen J, Siemons W, Verdonk R. Bicipitoradial bursitis. A case report. Acta Orthop Belg. 2006;72:362-5.

3. Behun MA, Geeslin AG, O'Hagan EC, King JC. Partial tears of the distal biceps brachii tendon: a systematic review of surgical outcomes. J Hand Surg Am. 2016;41(7):e175-89.

4. Kelly MP, Perkinson SG, Ablove RH, Tueting JL. Distal biceps tendon ruptures: an epidemiological analysis using a large population database. Am J Sports Med. 2015;43(8):2012-7.

5. Sutton KM, Dodds SD, Ahmad CS, Sethi PM. Surgical treatment of distal biceps rupture. J Am Acad Orthop Surg. 2010; 18(3):139-48.

6. Dillon MT, Bollier MJ, King JC. Repair of acute and chronic distal biceps tendon ruptures using the EndoButton. Hand ( $\mathrm{N}$ ). 2011;6(1):39-46.

7. Furia JP, Rompe JD, Maffulli N, Cacchio A, Schmitz C. Radial extracorporeal shock wave therapy is effective and safe in chronic distal biceps tendinopathy. Clin J Sport Med. 2017; 27(5):430-7.

8. Barker SL, Bell SN, Connell D, Coghlan JA. Ultrasound-guided platelet-rich plasma injection for distal biceps tendinopathy. Shoulder Elbow. 2015;7(2):110-4.

9. Bourne $\mathrm{MH}$, Morrey BF. Partial rupture of the distal biceps tendon. Clin Orthop Relat Res. 1991;(271):143-8.

10. Frazier MS, Boardman MJ, Westland M, Imbriglia JE. Surgical treatment of partial distal biceps tendon ruptures. J Hand Surg Am. 2010;35(7):1111-4.

11. Bain Gl, Johnson LJ, Turner PC. Treatment of partial distal biceps tendon tears. Sports Med Arthrosc Rev. 2008;16(3):15461.

12. Festa A, Mulieri PJ, Newman JS, Spitz DJ, Leslie BM. Effectiveness of Magnetic resonance imaging in detecting partial and complete distal biceps tendon rupture. J Hand Surg Am. 2010;35(1):77-83.

13. Failla JM, Amadio PC, Morrey BF, Beckenbaugh RD. Proximal 
radioulnar synostosis after repair of distal biceps brachii rupture by the two-incision technique. Report of four cases. Clin Orthop Relat Res. 1990;(253):133-6.

14. Morrey BF, Askew LJ, An KN, Dobyns JH. Rupture of the distal tendon of the biceps brachii. A biomechanical study. J Bone Joint Surg Am. 1985;67(3):418-21.

15. Lee HG. Traumatic avulsion of tendon of insertion of biceps brachii. Am J Surg. 1951;82(2):290-2.
16. Failla JM, Amadio PC, Morrey BF. Post-traumatic proximal radio-ulnar synostosis. Results of surgical treatment. J Bone Joint Surg Am. 1989;71(8):1208-13.

17. Bisson L, Moyer M, Lanighan K, Marzo J. Complications associated with repair of a distal biceps rupture using the modified two-incision technique. J Shoulder Elbow Surg. 2008;17(1 Suppl):67-71S. 